\title{
Modified Approach for VSS NLMS Algorithm for Echo Cancellation in Teleconference System
}

\author{
T.Suneetha \\ Assistant professor \\ Department of E.C.E \\ R.V.R\&J.C College of \\ Engineering, Guntur
}

\author{
A.Hareesh \\ Student (M.Tech) \\ Department of E.C.E \\ R.V.R\&J.C College of \\ Engineering, Guntur
}

\author{
G.Sudhavani \\ Associate professor \\ Department of E.C.E \\ R.V.R\&J.C College of \\ Engineering, Guntur
}

\begin{abstract}
Mobile communication or wireless phones have become need of everyone in this technological era of global communication. Wireless phones not only provide voice communication, they can also easily be used for other services. But voice quality is always preferable over other services of cellular networks. This demand of superior voice quality, just as like as wired line, over a wireless network has developed and is known as echo cancellation. The main aim of the acoustic echo cancellation is to remove the echoes present in desired signal and to provide echo free speech to the listeners. In this paper, a new approach for acoustic echo cancellation [1] and double talk detection for a teleconference system in non stationary environmental conditions is proposed. In this approach the impulse response of the loud speaker is assumed to be available and is estimated from the digital low pass filter. This approach is more common in the sense that it is applied to all existing algorithms. Performance evaluation of the proposed algorithm is known by calculating echo return loss enhancement (ERLE). This approach shows better performance for non stationary environment and reduces the complexity of both acoustic echo cancellation and double talk detection algorithms and this can be implemented with the help of MATLAB.
\end{abstract}

\section{Keywords}

Acoustic echo cancellation, Adaptive filtering, loud speaker impulse response, double talk detection.

\section{INTRODUCTION}

The communication technique has been changed a lot in recent years. Hands free communication had become more popular in modern telecommunication. Now a day's people are more interested in hands free speech telephony with enclosed loud speaker and microphone instead of wired telephony. However the presence of acoustic coupling between loudspeaker and microphone, the system would produce a loud echo that will cause the conversation difficult. The main aim of any service provider is to provide good voice quality. So, in order to provide good quality of speech it is necessary to remove the undesired echo signal in original speech signal. For this the echoes can be removed by two ways. One is echo suppression and another one is echo cancellation. But unfortunately echo suppression is not possible for full duplex communication; this is only works good for half duplex communication. However the full duplex communication is more needed in real time applications. This leads to the development of echo cancellers.

Acoustic echo cancellation (AEC) in teleconference system is still one of the active fields of research. The need for acoustic echo cancellation is to remove the echoes which are occurring due to the acoustic coupling between the micro phone and loud speaker in a communication system. The typical set up for acoustic echo cancellation is shown below Fig 1 .

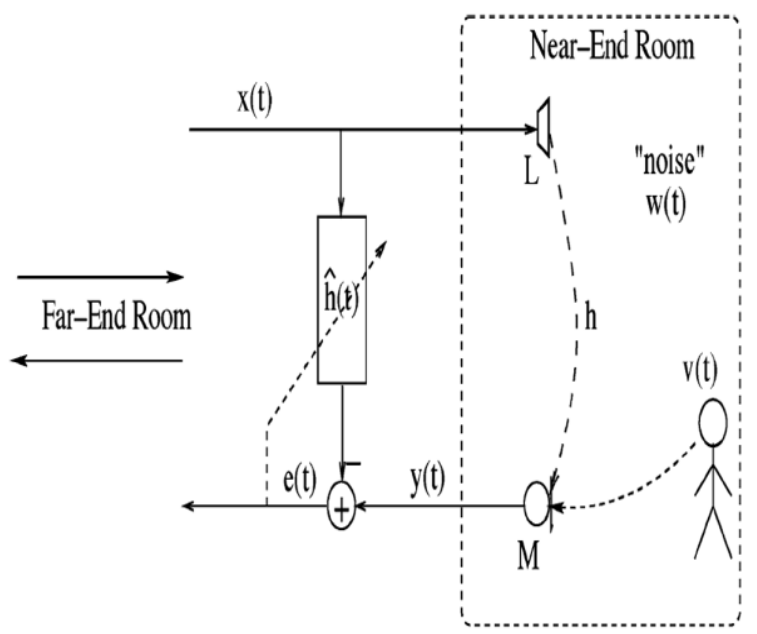

Fig 1: Typical set up for AEC

From the above typical set up the near end speech signal $\mathrm{v}(\mathrm{t})$ is picked up by the micro phone $\mathrm{M}$ and is propagated through the channel and is emitted through loud speaker L into the near end room. During the double talk which is the case where the near end speech signal and far end speech signal is present , the near speech signal at the of the micro phone is corrupted by far end speech signal $x(t)$ that is propagated from loud speaker to micro phone in the near end room. The resulting signal at the output of micro phone during double talk is

$y(t)=h^{T} X(t)+v(t)+w(t)$

Where $\mathrm{h}$ is the impulse response of the near room, w (t) is noise present in the near end room and $\mathrm{X}(\mathrm{t})$ is input data vector is defined as follows

$X(t)=[x(t) x(t-1) \ldots x(t-N+1)]^{T}$

Where $\mathrm{n}$ is the order of the impulse response and is modeled as FIR filter because when we use higher order filters the computational complexity of IIR filters increases. And h can be defined as

$h=\left[\begin{array}{llll}h_{0} & h_{1} & \ldots & h_{n-1}\end{array}\right]^{T}$

In real time the impulse response is time varying in nature so in order to adapt to these variations the estimate these variations is considered to cancel the echoes. This can be 
estimated by using the adaptive filters [2]. Let $h^{\wedge}$ denotes estimate of the impulse response. From this the error signal can be obtained as

$e(t)=y(t)-\hat{\mathrm{h}}^{T}(t) x(t)$

In ideal conditions above equation equal to $\mathrm{v}(\mathrm{t})$ and here it is assumed that $\mathrm{h}(\mathrm{t})$ and $\hat{\mathrm{h}}(\mathrm{t})$ are of same lengths.

Suppose when no near end speech signal is present the e $(\mathrm{t})$ can be used to adapt $\hat{h}(t)$ by using any one of the adaptive filtering algorithms. The most general one is normalized least mean square algorithm (NLMS) which is shown to be performing well for these AEC problems. And in this paper the variable step size parameter for adaptation of filter for non stationary environment presented. So with this step size adaptation loss of the desired signal can be reduced when compared to the fixed step size value.

When double talk is occurred, however the near end speech signal v ( $t$ ) can cause the adaptive filter to diverge. So during the double talk we need to stop the filter adaptation. This can be done by using the double talk detection (DTD) [3] algorithms. In this approach normalized cross correlation (NCR) algorithm for double talk detection is used, based on the decision of NCR algorithm the filter adaptation can be stopped. In this paper the combination of the both adaptive filtering algorithm and double talk detection algorithm is used and implemented as one for real time application. And performance of this approach is compared with other existing algorithms. And it could be shown that by using this impulse response measurement application to these algorithms increase the computational efficiency and with improved performance.

In this paper the loud speaker impulse response measurement (LIME) approach [4] to AEC and DTD algorithms has been applied. The LIME approach for the DTD is denoted as DTDLIME approach and LIME approach for AEC is denoted as AEC-DTD. The approach is based on the fact that all far end speech signal and no near end speech signal is filtered by the time invariant impulse response for the loud speaker L in fig: 1.and this can be exploited and with known impulse response it can modify the many existing AEC and DTD algorithms

\section{APPLIATION OF LIME APPROACH TO AECAND DTD ALGORITHMS}

\subsection{ACE- LIME Approach}

The loud speaker impulse response $\mathrm{h}$ in (1) includes the both the impulse response of the echo path $h_{E}$ which is time varying and time invariant impulse response of the loud speaker $h_{L}$. This can be written as

$h=h_{E} * h_{L}$

Where * denotes the convolution operation and here the assumed impulse response can be approximated as linear. Therefore the $h_{L}$ and $h_{E}$ can be expressed as

$h_{L}=\left[\begin{array}{llll}h_{L, 0} & h_{L, 1} & \ldots & h_{L, p-1}\end{array}\right]^{T}$

$h_{E}=\left[h_{E, 0} h_{E, 1}, \ldots h_{E, m-1}\right]^{T}$

Where $\mathrm{p}$ denotes the length of $h_{L}$ and $\mathrm{m}$ denotes the length of $h_{E}$. Therefore the length of impulse response from (5) is given by

$n=p+m-1$

Since most of the AEC's work with the model shown in (1) and this can be rewritten as
$y(t)=h_{E}^{T} \bar{X}(t)+v(t)+w(t)$

It is known that the impulse response $h_{L}$ is known. And $\bar{X}(t)$ can be expressed as

$\bar{X}(t)=[\bar{x}(t) \bar{x}(t-1) \ldots \bar{x}(t-n+1)]^{T}$

From the above equation (9) is almost equal to (1) and the length $h_{E}$ is less than $\mathrm{h}$ and computational complexities of AEC filter adaptation algorithms depends on length of the adaptive filter to estimate. From the above equations (1) through (10) shows the reduction of computational complexity of AEC adaptation filter. It should be noted that this reduction is possible only when there is a good estimate of the $h_{L}$ is available.

\subsection{AEC adaptation algorithm}

In this paper the NLMS algorithm [5] with variable step size is proposed. The main purpose of this algorithm is for proper convergence rate of the filter and adaptability of step size to the variable environmental conditions. This will work well for the non stationary environment [6] when compared to the fixed step size parameter. In this NLMS algorithm the weight updating vector is given by

$w(t+1)=w(t)+\mu e(t) X^{*}(t)$

Where $\mu$ is step size parameter and this can calculated as

$\mu=\frac{\beta}{c+X(t) X^{*}(t)}$

Here the $\beta$ is normalization factor and $\mathrm{c}$ is small positive constant to avoid divide by zero condition. In this approach the step size parameter is varying according to the signal value instantly. This is given as

$$
\begin{aligned}
& \mu=\frac{1}{X(t) X^{T}(t)}\left[1-\sqrt{\frac{E\left\{y^{2}(t)\right\}-E\left\{\hat{y}^{2}(t)\right\}}{E\left\{e^{2}(t)\right\}}}\right] \\
& \mu=\frac{1}{X(t) X^{T}(t)}\left[1-\sqrt{\frac{\sigma_{y}^{2}(t)-\sigma_{\hat{y}}^{2}(t)}{\sigma_{e}^{2}(t)}}\right]
\end{aligned}
$$

Where $E\{\cdot\}$ denotes the expectation value and $\hat{y}(t)$ is out put expectation value. And $\mu$ value can be varied as

$\mu=\left\{\begin{array}{c}\mu_{N L M S}: \text { for } N \leq L \\ \frac{1}{X(t) X^{T}(t)}\left[1-\sqrt{\frac{E\left\{y^{2}(t)\right\}-E\left\{\hat{y}^{2}(t)\right\}}{E\left\{e^{2}(t)\right\}}}\right]: \text { otherwise }(15)\end{array}\right.$

From above $\mathrm{N}$ is the length of the adaptive filter; $\mathrm{L}$ is the length of impulse response.

\subsection{DTD-LIME Approach}

In this section the application of the DTD algorithm to LIME approach is presented. Most of DTD algorithms work well with the data model shown in (1) and $y(t)$ is filtered version of the room impulse response $h$. In this DTD-LIME approach the data model (1) can be modified as

$y(t)=h_{L}^{T} \tilde{X}(t)+v(t)+w(t)$

Where

$\tilde{X}(t)=[\tilde{x}(t) \tilde{x}(t-1) \ldots \tilde{x}(t-N+1)]$
$\tilde{x}(t)=\hat{h}_{E}^{T} \check{x}(t)$
$\check{x}(t)=[\check{x}(t) \check{x}(t-1) \ldots \tilde{x}(t-N+1)]$

The computational complexities of many DTD algorithms are generally proportional to the length of the filter AEC model. By applying the DTD algorithm to model (16) instead of (1) 
reduces the computational complexity of DTD algorithm to the great extent. In this paper the NCR algorithm for double talk detection is efficiently used and it shows better results as performance wise when compared to other algorithms.

\subsection{DTD detection algorithm}

This section describes the NCR algorithm for DTD. In NCR algorithm the cross correlation between error signal $e(t)$ and micro phone signaly $(t)$ had been taken as $r_{e y}$ and is given by

$r_{e y}=E\{e(t) y(t)\}$

However the normalized decision static variable $\xi_{N C C}$ from equation (15) and (20) can be defined as

$\xi_{N C C}=1-\frac{\hat{\mathrm{r}}_{\mathrm{ey}}}{\hat{\sigma}_{\mathrm{y}}^{2}}$

For real time applications $\hat{\sigma}_{\mathrm{y}}^{2}$ and $\hat{\mathrm{r}}_{\mathrm{ey}}$ can be chosen as estimated values because actual values are not known exactly. For good adaptive algorithm the adaptive filter converges to an echo path $\hat{h}$ to approximately same length as h. thus finally the double talk detection decision can be made as

Decision $=\left\{\begin{array}{c}\xi_{N C C}(t) \leq T: \text { talk present } \\ \xi_{N C C}(t)>T: \text { no talk }\end{array}\right.$

And $\mathrm{T}$ is small threshold value and it should be less than or equal to 1 .

\section{LOUD SPEAKER IMPULSE RESPONSE MEASUREMENT}

The impulse response of the loud speaker is measured in different ways. The best and most direct way is compute it from the measurements made in the anechoic chamber. However methods computing the impulse response are directly taking the measurements of echoic room. If this impulse response is time varying this LIME approach is not suitable. Fortunately, it seems that loud speaker impulse response is relatively time-in varying in nature at least for some sophisticated speakers.

Indeed this time invariance property is one which is used by the music products such as Dirac research corrector that can compensate for the acoustic properties of loud speaker.
However it is clear that loud speaker impulse response is highly directional dependent but this is approach is concentrating on the part which is directional independent (same for the case of Dirac research corrector).

\subsection{Acoustic echo path changes adaptation}

Most of the DTD algorithms encounters problems during the changes occurred between the loud speaker and micro phone. However this DTD-LIME approach is sensitive to these small variations and this can be observed in model (18). Suppose if $h_{E}$ is changes more rapidly this all these model are not working properly. So one way to avoid this is use the adaptive filter estimate just before the double talk occurs to cancel the echo and continue to adapt filter during double talk and use most recent adaptive estimate in the DTD.

\subsection{Performance measures}

The echo return loss enhancement is used to measure the performance of the AEC. And is defined as the

$\operatorname{ERLE}(t)=-10 \log \left(\frac{E\left[e^{2}(t)\right]}{E\left[y^{2}(t)\right]}\right)$

And measurement of the mean square error of the microphone signal is also measured.

\section{SIMULATION RESULTS}

The model shown in Fig 1 is used to generate the data. The impulse response $\mathrm{h}$ is measured in the general auditorium using AEC set up with known $h_{L}$.for keeping the computational complexity of NCR algorithm low, this approach considering the input signal sampling frequency as $16 \mathrm{KHz}$ (this can allowed by using the shorter impulses there by reducing the computational complexity). The NLMS algorithm for AEC performs well for both with and without LIME approaches but it improves performance with reduced computational complexity [7] by using LIME approach. Fig 2 \&3 shows the simulation results of DTD and ERLE for ACE with and with out LIME approach and these are simulated by using the MATLAB [8] and then these are compared ordinary NLMS algorithm. In this approach a 512 order adaptive filter is used for estimation of echo signal. For double talk detection the threshold value $\mathrm{T}$ as 0.95 is used.

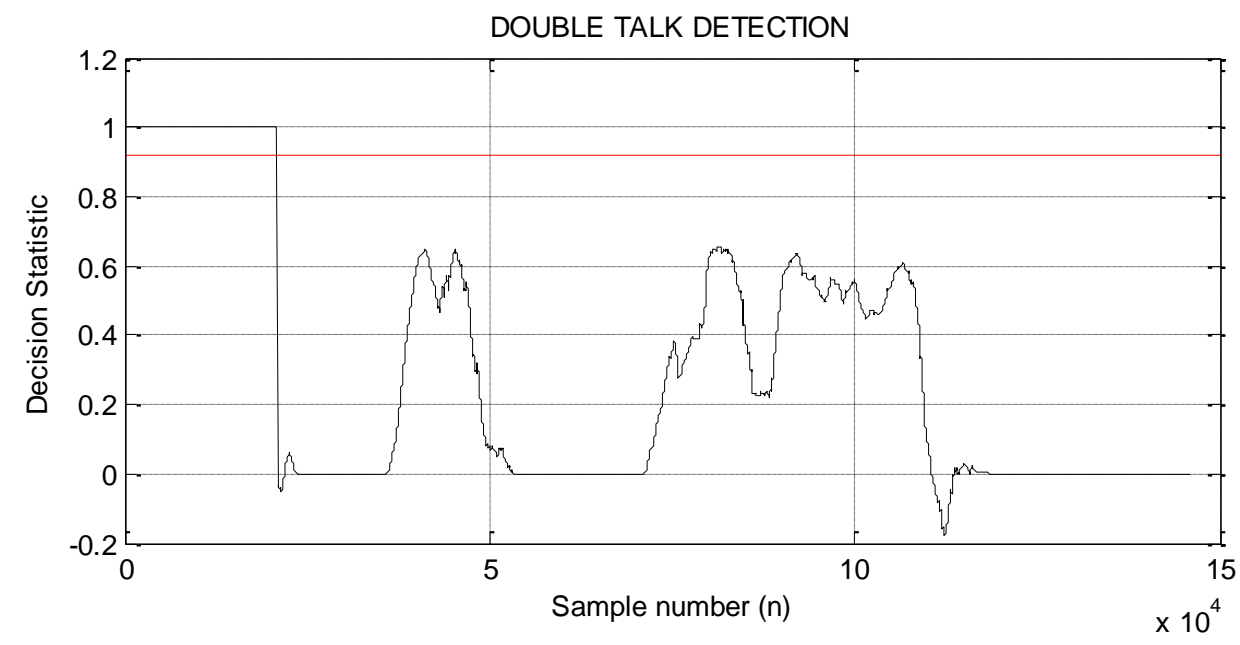

(a) 


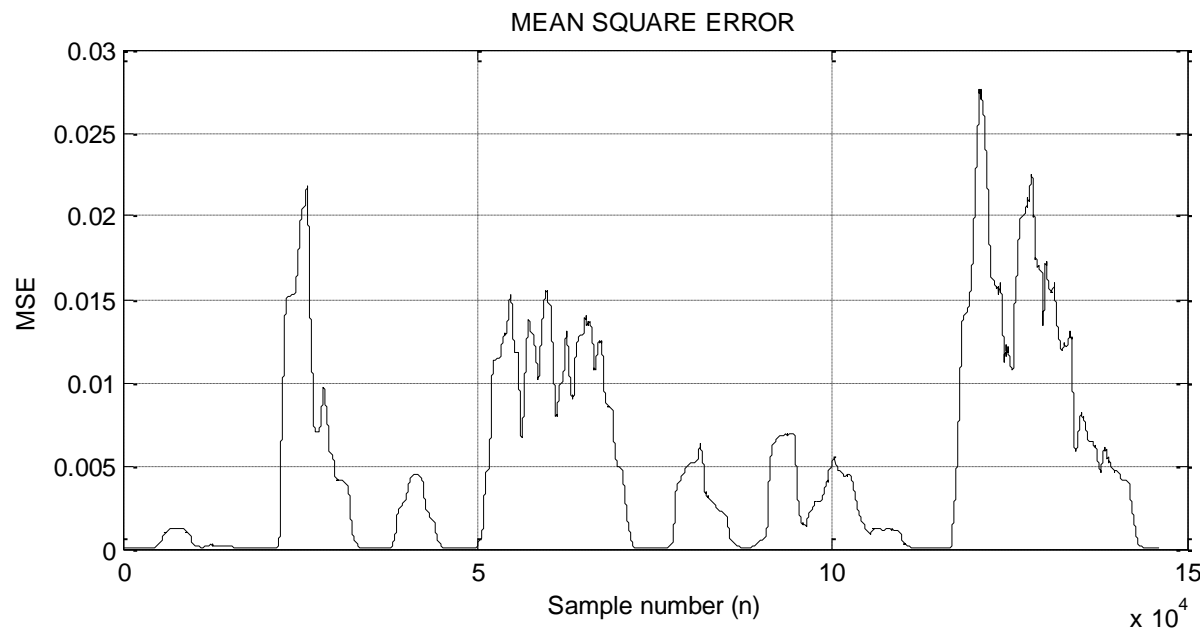

(b)

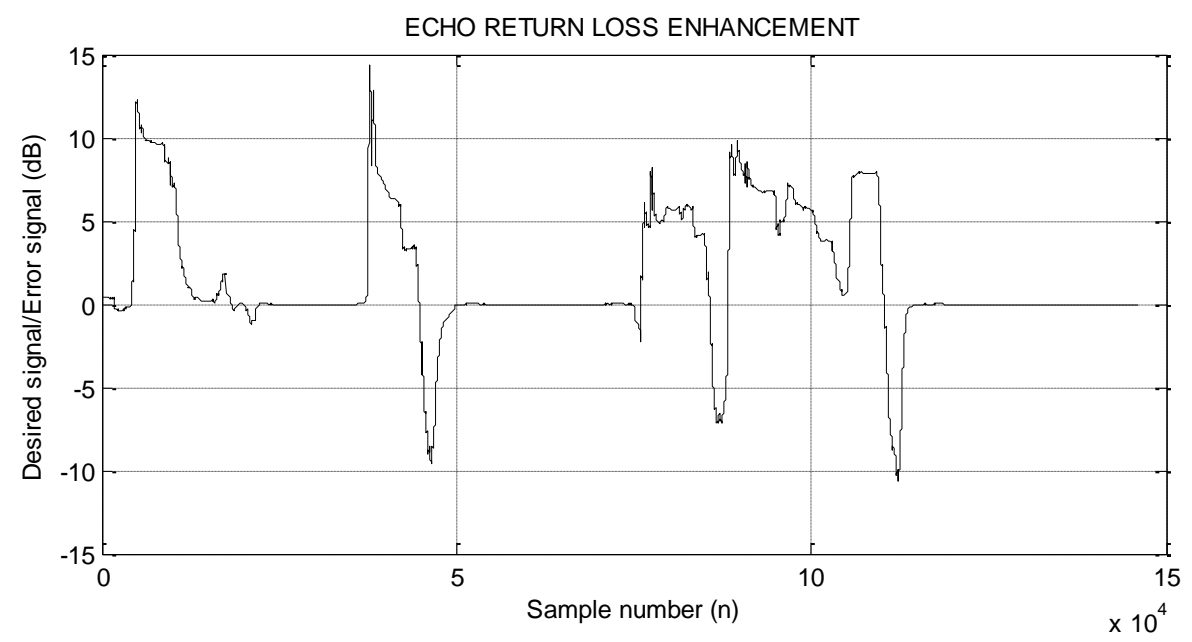

(c)

Fig 2: Illustrations of DTD and ERLE for AEC without LIME approach, (a) Double talk detection, (b) Mean square error,

(c) Echo return loss enhancement

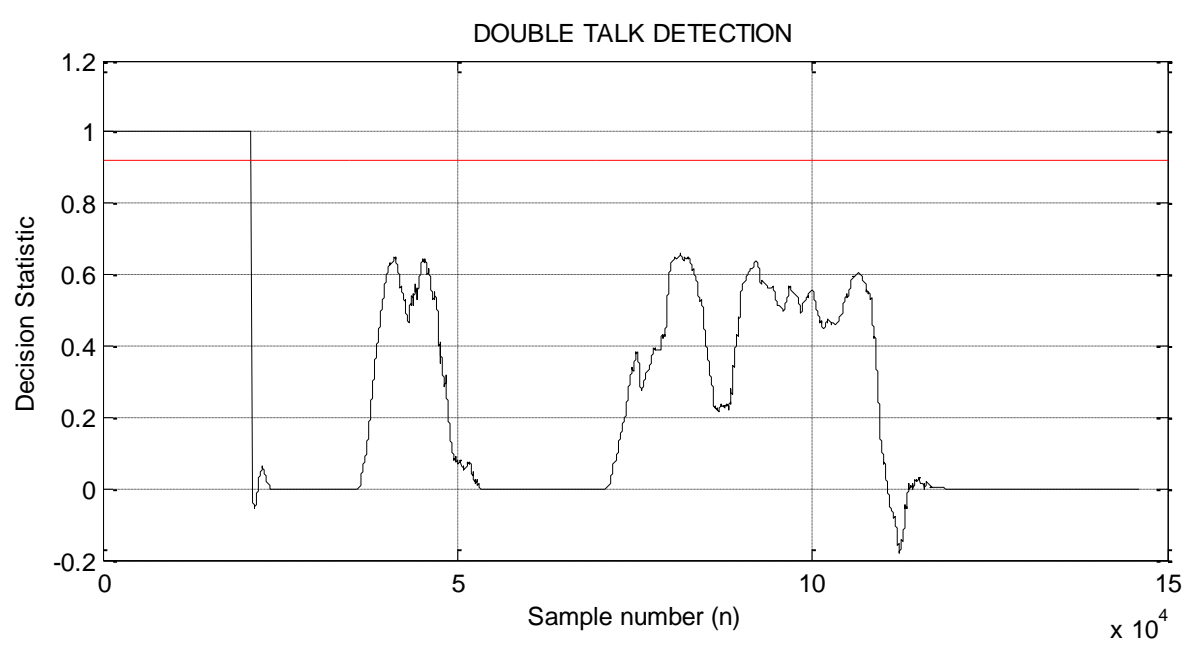

(a) 


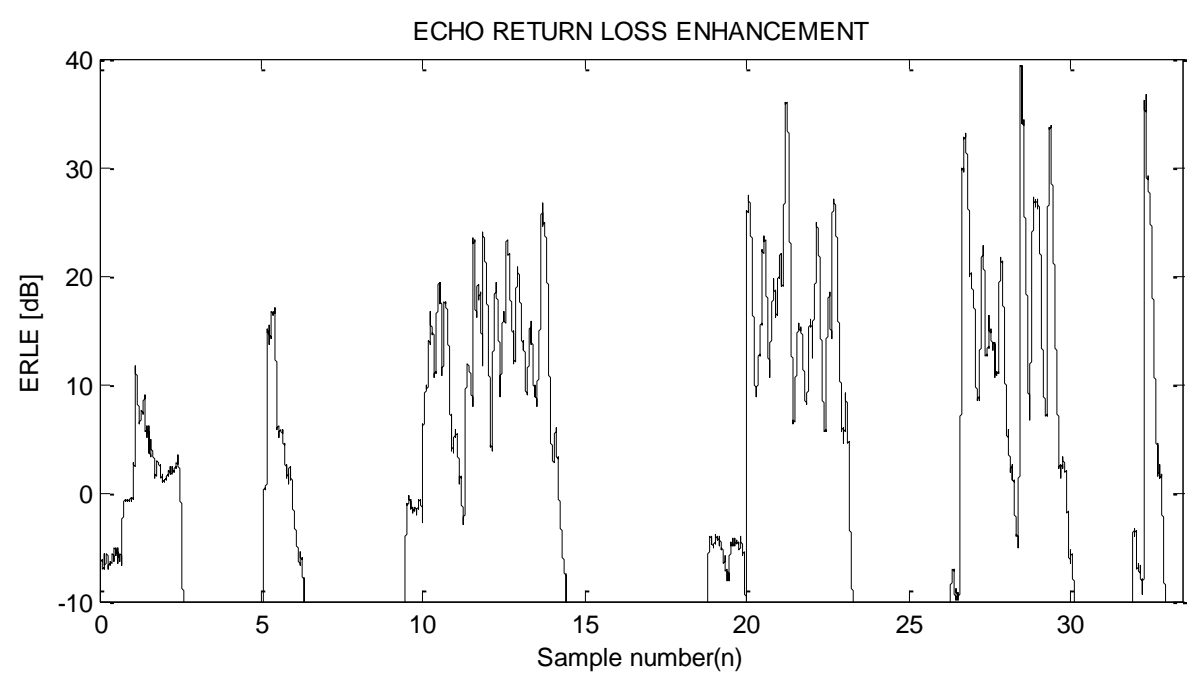

(b)

Fig 3: Illustrations of DTD and ERLE for AEC with LIME approach, (a) Double talk detection,

(b) Echo return loss enhancement

From the Fig $2 \& 3$ it is observed that the performance of the AEC is improved a lot it shows that ERLE of nearly $40 \mathrm{~dB}$ with LIME approach which is $20 \mathrm{~dB}$ more than the ordinary NLMS algorithm without LIME approach. In case of DTD it gives almost gives the equal results because when using higher order filters only minute variations will be nullified for fast convergence rate of the filter.

\section{CONCLUSION}

In this paper a modified approach of NLMS algorithm with variable step size for echo cancellation in a teleconference system for non stationary environment is presented. This is implemented for only single channel. For further work it may also possible for multichannel or a stereo outputs.

However from the observations of simulation results the AEC-LIME approach gives the good satisfactory results for

non stationary environments with variable step size and ERLE will be more than the ordinary NLMS algorithm [9] with fixed step size value and computational complexity of NCR algorithm for DTD by using this approach is reduced more.

\section{REFERENCES}

[1] M.M. Sondhi, 1967, "An adaptive echo canceller", Bell syst.Tech J., Vol.XLVI, no.3, pp.497-510.

[2] C.Breining, P.Dreiseited, E.Hansler, A.Mader, B.Nitsch, H.Puder, T.Schertler, G.Schmidt and J.Tilp, 1999, "Acoustic echo control- an application of very high order adaptive filters", IEEE Signal Process.Mag., Vol.16, no. 4 , pp. $42-69$
[3] J.H.Cho, D.P. Morgan, and J.Benesty, 1999, “An objective technique for evaluating double talk detectors in acoustic echo cancellers", IEEE trans. Speech audio process, Vol.7, no.6, pp.718-724.

[4] P.Ahgren and P.Stoica, 2003, "A Simple method for estimating the impulse responses of loud speakers", IEEE Trans. consumer elect. Vol.49, no.4, pp.889-893.

[5] Constsntain paleologu, Member, IEEE, Silviu ciochina, Member, IEEE, and Jacob Benesty, Senior Member, IEEE,2008, "Variable step size NLMS algorithm for under modeling Acoustic Echo Cancellation", IEEE signal proc.,vol.15.

[6] M.Niedwiecki, 2000, "Identification of time varying process", John Wiley and sons, Ltd.

[7] Pankush Singla, Sandeep Kaur, Sukhpreet Singh,2013, “A Review on Acoustic echo Cancellation Techniques", International Journal of Advanced Research in Computer Engineering \& Technology (1278-1323),Vol.2,Issue.11.

[8] Rohit Srivastava, Dheeraj Kumar Singh, Jwalant Baria, 2012, "Mat lab Implementation of Echo Cancellation in Telephone Line", International Journal of Emerging Technology and Advanced Engineering (2250-2459), Vol. 2, Issue 10.

[9]Ragahava Yathiraju, 2013, "Acoustic Echo cancellation Using Conventional Adaptive Algorithms", International Journal of Inventive Engineering and Science (2319. 9598), Vol.1, Issue.12. 